\title{
Pseudo-aneurisma em tubo valvulado de pericárdio bovino corrugado após reconstrução da aorta ascendente: relato de caso
}

\author{
Noedir A. G. STOLF*, Carlos M. A. BRANDÃO*, Cristiano N. FABER* ${ }^{*}$ Paulo M. PÊGO- FERNANDES*, \\ Roberto COSTA* ${ }^{*}$ Adib D. JATENE*
}

RBCCV 44205-476

Stolf N A G, Brandão C M A, Faber C N, Pêgo-Fernandes P M, Costa R, Jatene A D - Pseudo-aneurisma em tubo valvulado de pericárdio bovino corrugado após reconstrução da aorta ascendente: relato de caso. Rev Bras Cir Cardiovasc 1999; 14 (4): 340-3.

RESUMO: Os autores descrevem o caso de paciente que, 9 anos após a correção cirúrgica de um aneurisma de aorta ascendente com tubo valvulado de pericárdio bovino corrugado, evoluiu com a formação de um pseudo-aneurisma de aorta localizado, posteriormente, sobre a linha de sutura do tubo de pericárdio bovino. Foi realizada substituição do tubo de pericárdio bovino por tubo de Dacron valvulado (com prótese mecânica) e reimplante dos óstios coronários utilizando-se a técnica de hemi-Cabrol. O tempo de seguimento pós-operatório do paciente é de 12 meses, permanecendo assintomático.

DESCRITORES: Prótese vascular. Bioprótese. Falso aneurisma, cirurgia. Aneurisma aórtico, cirurgia.

\section{INTRODUÇÃO}

A correção dos aneurismas da aorta ascendente durante muitos anos representou um grande desafio aos cirurgiões. Muitas das dificuldades foram amenizadas após a introdução da técnica cirúrgica proposta por BENTALL \& DE BONO (1), em 1968, que consistia na troca da aorta ascendente acompanhada do reimplante dos óstios coronários. Para tal substituição dispomos de um grande número de próteses tubulares valvuladas, de diversos materiais, sendo as mais comuns em nosso meio as próteses de Dacron e as de pericárdio bovino. O pericárdio bovino preservado em glutaraldeído tem sido usado em nosso meio desde 1979 para ampliações da aorta ${ }^{(2)}$. Posteriormente, iniciou-se a utilização de tubos de pericárdio bovino valvulados para a substituição da aorta ascendente, tendo como vantagens a flexibilidade e a maleabilidade do tecido na feitura das anastomoses, o baixo custo e a impermeabilidade ao sangue (dispensando a necessidade de pré- coagulação do tubo), diminuindo, assim, o sangramento pós-operatório (3-5). SALLES et al. (4) desenvolveram um tubo de pericárdio bovino corrugado, que apresenta uma sutura dupla de poliéster multifilamentado, o que permite que o tubo seja cortado sem que haja deiscência na linha de sutura, tendo, na época da publicação do trabalho, operado uma série de 10 pacientes portadores de aneurisma de aorta ascendente ou dissecção aórtica, onde se substituiu a aorta ascendente por este tubo, apresentando bom resultado em período médio de seguimento de 8 meses, não relatando, neste período, nenhum problema relacionado à prótese.

\footnotetext{
Trabalho realizado no Instituto do Coração do Hospital das Clínicas da Faculdade de Medicina da Universidade de São Paulo. São Paulo, SP, Brasil.

* Do Instituto do Coração do Hospital das Clínicas da Faculdade de Medicina da Universidade de São Paulo.

Recebido para publicação em maio de 1999.

Endereço para correspondência: Av. Dr. Eneás de Carvalho Aguiar, 44. Cerqueira César, São Paulo, SP, Brasil. CEP: 05403-000. Tel. (011) 30695318. e-mail:stolf@incor.usp.br
} 
Stolf N A G, Brandão C M A, Faber C N, Pêgo-Fernandes P M, Costa R, Jatene A D-Pseudo-aneurisma em tubo valvulado de pericárdio bovino corrugado após reconstrução da aorta ascendente: relato de caso. Rev Bras Cir Cardiovasc 1999;14 (4): 340 -3.

Como complicações mais freqüentes, independentemente do tipo de enxerto utilizado para a troca da aorta ascendente, encontramos o sangramento pós-operatório, infecção no enxerto, formação de falsos aneurismas nas anastomoses $(3,6)$. Os enxertos de pericárdio bovino têm sido utilizados em nosso meio. Pouco se sabe a respeito da evolução tardia dos enxertos de pericárdio bovino corrugado quando utilizados para a substituição da aorta ascendente. Os autores descrevem o caso de paciente operado por aneurisma de aorta ascendente onde foi utilizado um tubo valvulado de pericárdio bovino corrugado, que evoluiu com a formação de um pseudo-aneurisma, sendo necessária a sua substituição.

\section{RELATO DO CASO}

Paciente do sexo masculino, de 47 anos de idade, com história de aneurisma de aorta ascendente, operado em maio de 1989 no Instituto do Coração do Hospital das Clínicas da Faculdade de Medicina da Universidade de São Paulo, sendo realizada correção do aneurisma através da interposição de tubo de pericárdio bovino valvulado (com prótese biológica) em aorta ascendente (operação de Bentall e De Bono), apresentando boa evolução pós-operatória. Permaneceu assintomático após a operação, fazendo acompanhamento ambulatorial periódico devido à dislipidemia. Durante acompanhamento, notou-se alargamento do mediastino, com imagem sugestiva de aneurisma de aorta à radiografia de tórax (Figura 1). A tomografia computado-

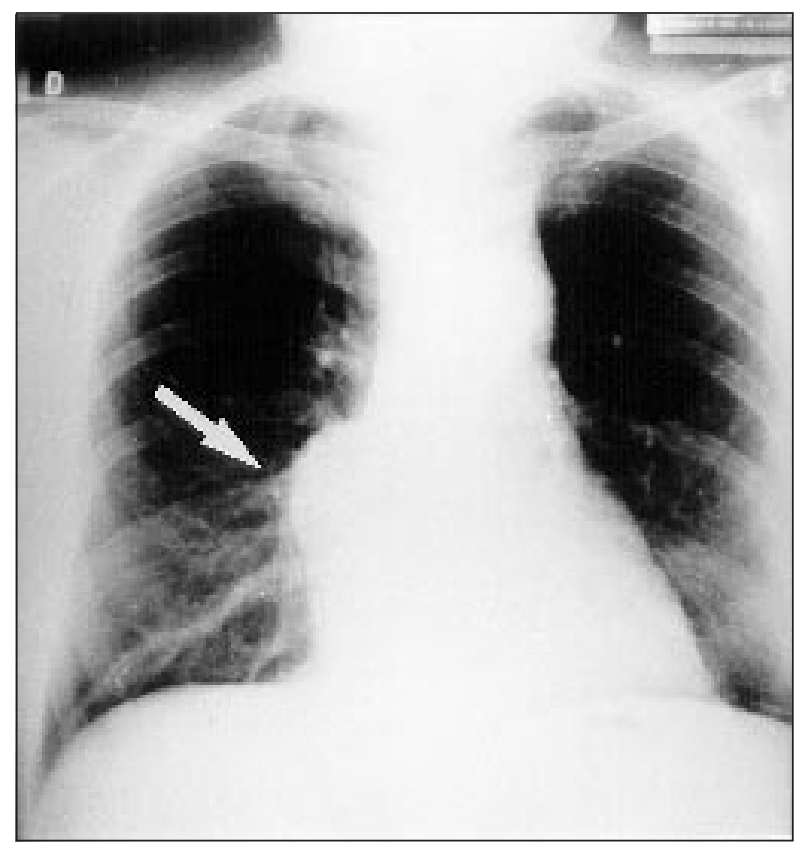

Fig. 1 - Radiografia de tórax mostrando alargamento do mediastino e imagem sugestiva de aneurisma de aorta (seta). rizada de tórax revelou aneurisma de aorta ascendente, sendo o diâmetro da porção inicial da aorta de $7,0 \mathrm{~cm}$ e o diâmetro de aorta ascendente de 7,9 $\mathrm{cm}$, joelho anterior e arco medindo $4,6 \mathrm{~cm}$ de diâmetro e presença de trombo mural (Figura 2). Em dezembro de 1997, foi realizado exame de ressonância magnética do tórax, que mostrou um pseudo-aneurisma da aorta 7,0 cm acima do plano valvar e arco aórtico proximal com dilatação (Figura 3).

Optou-se pelo tratamento cirúrgico, sendo realizado em janeiro de 1998, sob esternotomia mediana, com instalação de circulação extracorpórea (CEC), canulação da aorta e átrio direito, hipotermia moderada, utilizando-se solução cardioplégica sangüínea. $O$ achado intra-operatório foi de grande pseudo-aneurisma com trombos na sua capa. Desfeita a anastomose distal e aberto o tubo de pericárdio, visibilizaram- se os óstios coronários adequadamente implantados, sem deiscência. Havia uma abertura na parede posterior do tubo de pericárdio sobre a linha de sutura do tubo. Foi, então, retirada a prótese biológica, a que apresentava bom aspecto, e implantado um tubo de Dacron valvulado com prótese mecânica St Jude número 25. Para o reimplante das coronárias optou-se pela técnica de hemi-Cabrol, utilizando-se um tubo de PTFE número 10 para a anastomose da coronária esquerda, sendo a coronária direita anastomosada diretamente no tubo de Dacron, com bom resultado técnico. O tempo de CEC foi de $180 \mathrm{~min}$ e o tempo de pinçamento aórtico foi de $40 \mathrm{~min}$. O paciente evoluiu no pós-operatório, imediato com bloqueio atrioventricular total necessitando de implante de marcapasso cardíaco definitivo. Realizou- se exame de ressonância magnética no 9 dia de pós-operatório, que mostrou boas condições da correção cirúrgica. O paciente recebeu alta hospitalar no $18^{\circ}$ dia de pós-operatório, assintomático. O tempo de seguimento pós-operatório é de 12 meses e o paciente permanece assintomático.

\section{COMENTÁRIOS E CONCLUSÃO}

O manuseio cirúrgico do aneurisma da aorta ascendente, associado ou não à doença da valva aórtica, vem apresentando resultados progressivamente melhores devido aos avanços no diagnóstico, nos materiais e nas técnicas operatórias. O advento dos enxertos compostos (tubos valvulados) em 1968 (1) contribuiu também para diminuir a mortalidade operatória, bem como as complicações pós-operatórias (5-7). Entretanto, esta técnica pode apresentar complicações intra-operatórias de difícil controle, uma vez que o reimplante dos óstios coronários (realizado diretamente no tubo) pode levar à tração da parede da aorta a este nível e 


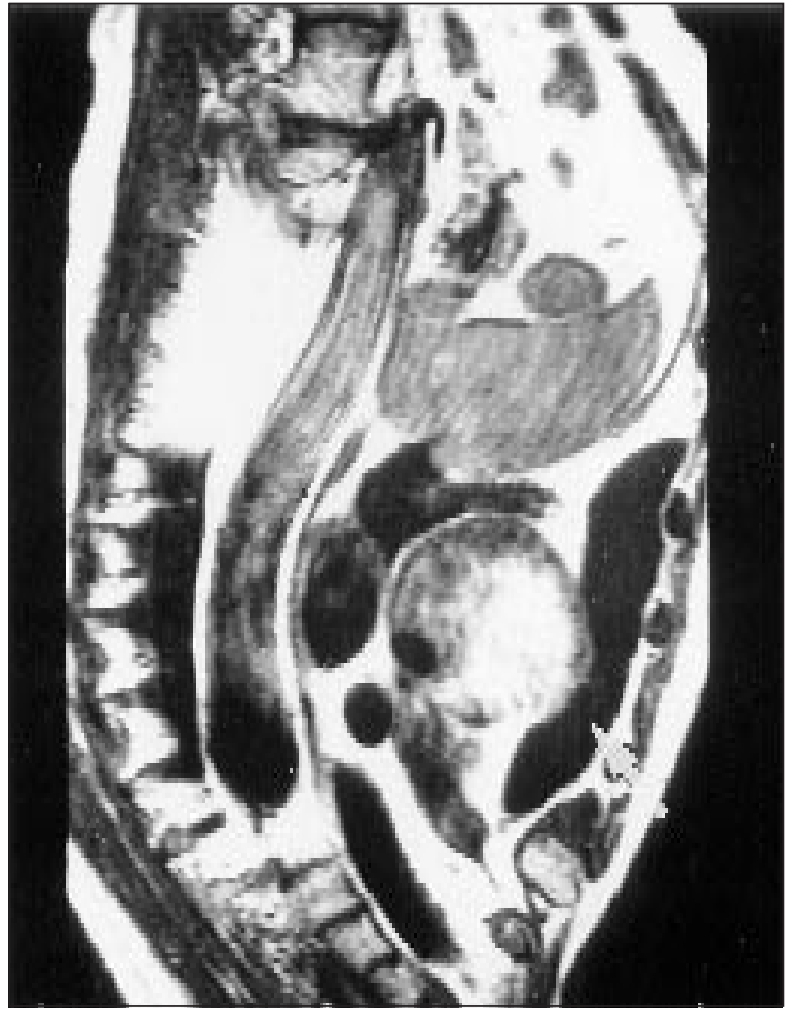

Fig. 2 - Tomografia computadorizada de tórax mostrando aneurisma de aorta ascendente e presença de grande trombo mural (seta).

tornar-se um local de sangramento de difícil acesso ${ }^{(6,8)}$. Este mecanismo parece ser o mesmo para a formação de pseudo-aneurismas de aorta que ocorrem tardiamente na zona do reimplante das coronárias, uma vez que é realizada a inclusão do tubo valvulado na capa do aneurisma e o sangramento pelas suturas levaria a um aumento da pressão na região ${ }^{(8,9)}$. As técnicas de reimplante dos óstios coronários com desinserção sem fechamento da capa do aneurisma foram propostas para evitar essa complicação. Outro ponto comum no aparecimento tardio de pseudo-aneurismas são as suturas, distal e proximal, entre a aorta e o tubo utilizado $(6,9)$. Entretanto, PÊGO-FERNANDES et al. (10) estudaram 38 pacientes, com um tempo de seguimento de 2 a 72 meses (média de 25 meses), em que esta técnica foi utilizada com bons resultados, sem complicações tardias.

Muitos materiais foram estudados no intuito de se encontrar o tecido ideal para a feitura das próteses, tais como Vinyon, Nylon, Orlon, Teflon e Dacron, sendo este, desde seu desenvolvimento, o material mais utilizado. A utilização do pericárdio bovino como material protético na correção de defeitos congênitos apresenta, como vantagens, menor custo, impermeabilidade, maleabilidade e flexibilidade do tecido, tornando as linhas de sutura

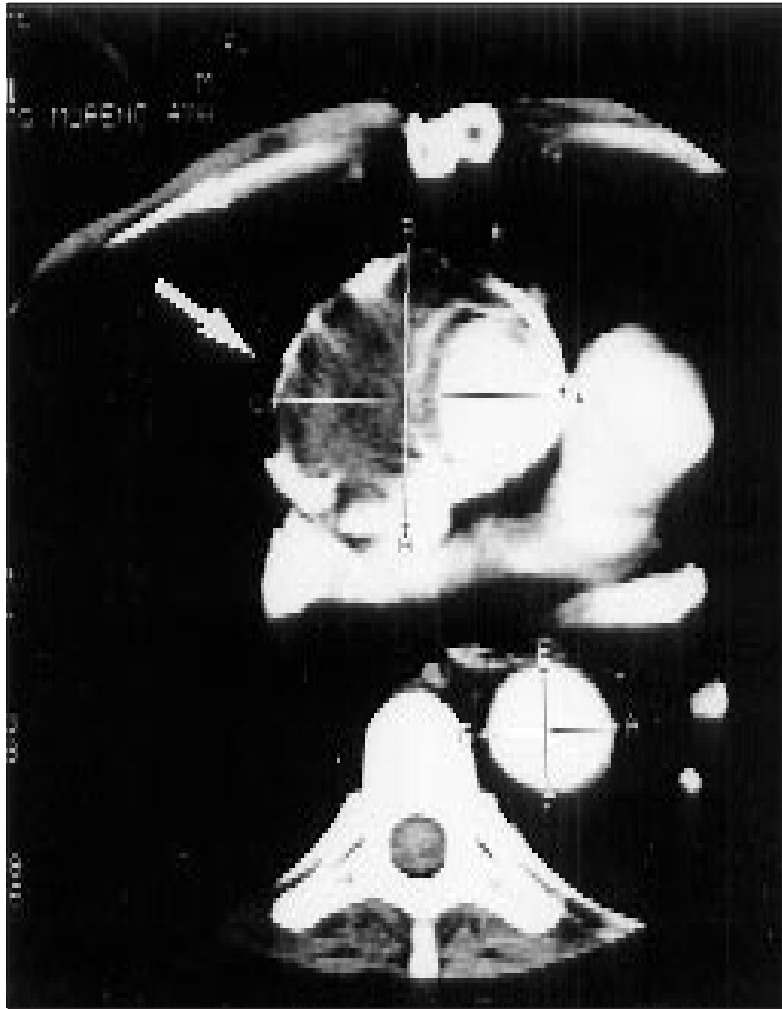

Fig. 3 - Ressonância magnética do tórax mostrando pseudo-aneurisma da aorta (seta).

mais hemostáticas $(2,3,11)$. SALLES et al. (4) desenvolveram um tubo corrugado de pericárdio bovino como alternativa ao Dacron. O pericárdio bovino, mesmo quando conservado em glutaraldeído, apresenta como maiores inconvenientes a calcificação e a formação de aneurismas a longo prazo (11-13). No entanto, KALIL et al. (14), avaliando tardiamente (tempo de seguimento de 2 a 7 anos) pacientes onde se utilizou o tubo de pericárdio para o tratamento de doenças da aorta, não relatam o aparecimento de calcificações ou pseudo-aneurismas, relatando, em $40 \%$ dos casos, dilatação moderada do enxerto.

Em nosso caso, a formação do pseudo-aneurisma ocorreu na linha de sutura da prótese, local não relacionado ao reimplante das coronárias ou anastomoses, fato este incomum.

Em conclusão, a utilização do pericárdio bovino como enxerto para o tratamento das afecções vasculares é alternativa viável, devendo-se lembrar que ainda faltam estudos com maior número de casos, bem como maior tempo de seguimento dos pacientes, a fim de que se demonstre com segurança a sua evolução a longo prazo. $O$ relato da complicação neste trabalho também constitui razão para cautela no seu uso extensivo. 
Stolf N A G, Brandão C M A, Faber C N, Pêgo-Fernandes P M, Costa R, Jatene A D - Pseudo-aneurisma em tubo valvulado de pericárdio bovino corrugado após reconstrução da aorta ascendente: relato de caso. Rev Bras Cir Cardiovasc 1999; 14 (4): 340-3.

RBCCV 44205-476

Stolf N A G, Brandão C M A, Faber C N, Pêgo-Fernandes P M, Costa R, Jatene A D - False aneurysm of crimped bovine pericardial conduit after reconstruction of the ascending aorta: case report. Rev Bras Cir Cardiovasc 1999; 14 (4): 340-3.

ABSTRACT: The authors describe the case of a patient who had an aneurysm of the ascending aorta repaired with biologic valved crimped bovine pericardial conduit. Nine years after the surgery he presented a false aneurysm of the ascending aorta, located posteriorly on the suture line of the pericardial tube. The patient was reoperated and the pericardial tube was replaced by a valved Dacron composite graft using the hemiCabrol technique for the reimplantation of the coronary ostia. At 12 months follow-up the patient remains free of symptoms.

DESCRIPTORS: Blood vessel prosthesis. Bioprosthesis. Aneurysm, false, surgery. Aortic aneurysm, surgery.

\section{REFERÊNCIAS BIBLIOGRÁFICAS}

1 Bentall $M$ \& De Bono $A$ - A technique for complete replacement of ascending aorta. Thorax 1968; 23: 338-9.

2 Braile D M, Bilaqui A, Ardito R V et al. - Alargamento da raíz de aorta com patch de pericárdio bovino preservado em glutaraldeído. Arq Bras Cardiol 1983; 41: 289-96.

3 Ardito R V, Santos J L V, Mayorquim R C et al. - Substituição completa da aorta ascendente e da valva aórtica com tubo valvulado de pericádio bovino. Rev Bras Cir Cardiovasc 1987; 2: 129-38.

4 Salles C A, Puig L B, Casagrande I S et al. - Early experience with crimped bovine pericardial conduit for arterial reconstruction. Eur J Cardio-thorac Surg 1991; 5: 273-9.

5 Bernardes R C, Reis Filho F A R, Marino R L et al. Surgical correction of aortic disease using intraluminal, crimped bovine pericardial graft. Ann Thorac Surg 1995; 60 (2 suppl): S316-21.

6 Kouchoukos N T, Marshall W G Jr, Wedige-Stecher T A - Eleven-years experience with composite graft replacement of the ascending aorta and aortic valve. J Thorac Cardiovasc Surg 1986; 92: 691-705.

7 Crawford E S, Svensson L G, Coselli J S, Safi H J, Hess $\mathrm{K} R$ - Surgical treatment of aneurysm and/or dissection at the ascending aorta, transverse aortic arch, and ascending aorta and transverse aortic arch: factors influencing survival in 717 patients. $J$ Thorac Cardiovasc Surg 1989; 98 (5 Pt1): 659-74.

8 Cabrol C, Pavie A, Mesnildrey P et al. - Long-term results with total replacement of the ascending aorta and reimplantation of the coronary arteries. $J$ Thorac Cardiovasc Surg 1986; 91: 17-25.

9 McFalls E O, Palac R, Gately H, Floten H S Pseudoaneurysm formation with superior vena caval syndrome 7 years after aortic composite graft placement. Ann Thorac Surg 1989; 48: 704-5.

10 Pêgo- Fernandes $P$ M, Stolf N A G, Beyruti R, Moreira L F P, Mady C, Jatene A D - Resultados da substituição da aorta ascendente e valva aórtica com reimplante de artérias coronárias. Arq Bras Cardiothorac 1990; 55: 361-5.

11 Gontijo B, Vrandecic M, Fantini F et al. - Replacement of ascending aorta and aortic arch with bovine pericardial patch: a preliminary report. Eur J Cardiothorac Thorac Surg 1995; 9: 127-32.

12 Bullaboy C A - Bovine pericardium: another cautionary note. Ann Thorac Surg 1989; 48: 743 (Letter).

13 Gabbay S, Bortollotti U, Factor S, Shore D F, Frater W $R$ - Calcification of implanted xenograft pericardium: influence of site and function. J Thorac Cardiovasc Surg 1984; 87: 782-7.

14 Kalil R A, Feldman C J, Ludwig F W et al. - Late evaluation of spiral computed tomography of smooth bovine pericardium grafts. Arq Bras Cardiol 1997; 69: 111-5. 